\title{
Equivalent Inertial Force Method of Seismic Calculation for Subway Station in Soft Site
}

\author{
Guofu Wang, ${ }^{1}$ Jinhua Shang, ${ }^{1}$ Xianfeng $\mathrm{Ma}^{2}$ and Xiyong Xu${ }^{1}$ \\ ${ }^{1}$ Jinan Rail Transit Group Co., Ltd., Jinan 250000, China \\ ${ }^{2}$ Department of Geotechnical Engineering, Tongji University, Shanghai 200092, China \\ Correspondence should be addressed to Jinhua Shang; jngjgcb2016@163.com
}

Received 20 July 2016; Revised 19 September 2016; Accepted 5 October 2016

Academic Editor: Matteo Aureli

Copyright (c) 2016 Guofu Wang et al. This is an open access article distributed under the Creative Commons Attribution License, which permits unrestricted use, distribution, and reproduction in any medium, provided the original work is properly cited.

\begin{abstract}
On the basis of the structural characteristics of subway stations, structural inertial force can be simplified as multiple concentrated force acting on the member connecting node by using equivalent inertial force method of seismic calculation in soft site, and then internal forces of the structure will be analyzed. According to the principle that the maximum bending moment values of equivalent inertial force method and dynamic time-history analysis method are equal and the location is the same, the value of equivalent inertial force is determined, and the shear and axial force are ensured by introducing correction factor. The result shows that the calculation results of equivalent inertial force method are accurate and reliable. It can meet the design requirements and improve computational efficiency.
\end{abstract}

\section{Introduction}

With the number of underground works and the frequency of seismic damage increase, especially serious damage of the subway station and tunnel caused by Kobe earthquake in Japan in 1995, more and more attention has been paid to the seismic performance of subway station structures. The subway station as a passenger waiting and transfer area, once destroyed in the earthquake, will result in significant economic losses because of high repair cost [1-3]. Therefore, it is necessary to conduct the study of subway station seismic design calculation method.

Domestic and foreign scholars have conducted depth studies on the seismic response of underground structures as well as failure mechanism $[2,4,5]$, especially in experimental research and numerical simulation [6-12]. Dynamic timehistory analysis method that is used in soft soil layer seismic response analysis of underground structures is highly consistent with the actual situation and calculates accurately, and so forth; however, the calculation process is complex, inefficient, and unsuitable for promotion. For soft soil stations, on the basis of the calculation of the results of dynamic time-history method, to establish equivalent inertial force method which is suitable for subway station seismic calculation of engineering design is an effective mean to improve computational efficiency.

\section{Fundamental}

Real inertial force under earthquake action, the same with gravity, is physical strength, which is applied to the structure according to the density distribution of the component. The form of inertial force distribution, value of inertial force, and constitutive model of soil and structure need to be determined in equivalent inertial force method. When the dynamic response of the subway station is analyzed by using equivalent inertial force method, it is necessary to make the appropriate assumptions to simplify the calculation model which has only force in the balance system. Seismic load and foundation soil resistance in equivalent inertial force are determined in the following two assumptions: (1) after the earthquake, the inertial force generated by the action of seismic waves in the subway stations is active force, whose value is determined by the size of the structure depth, seismic intensity, foundation soil properties, and other factors; (2) the movement and deformation of subway station are under 


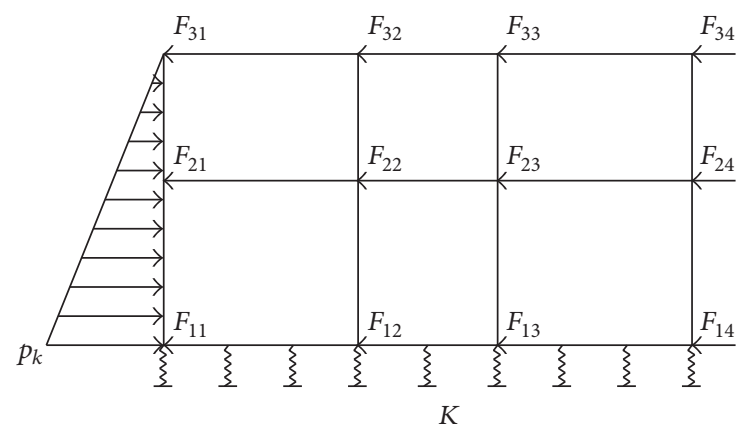

FIGURE 1: Distribution and calculation diagram of seismic load in subway station structure.

TABLE 1: Earthquake acceleration basic value $\left(k_{s}\right)$.

\begin{tabular}{lcccc}
\hline Fortification intensity (degree) & 6 & 7 & 8 & 9 \\
\hline$k_{s}(\mathrm{~g})$ & 0.05 & $0.10(0.15)$ & $0.20(0.30)$ & 0.40 \\
\hline
\end{tabular}

the function of restraints from surrounding soil, which is applied to the structure in the form of strata resistance. Strata resistance is passive force, whose value and distribution form are determined by the equilibrium of forces and mechanical characteristics of the structure.

\section{Establishment of Equivalent Inertial Force Method}

Due to the complication of internal components in subway station structure, it is difficult to exert inertial force according to the actual situation. Thus, based on the structural characteristics of the subway station, the inertia force can be simplified to multiple concentration forces and act on the node connected to the components, as shown in Figure 1. $F_{i j}$ is the equivalent horizontal seismic inertia force acting on node $i j$, which can be calculated by formula (1); $p_{k}$ is the maximum value of the triangle horizontal resistance, which can be calculated by the equilibrium condition of seismic load in horizontal direction; $K$ is the coefficient of subgrade reaction, which can be referenced to [13].

Take three-level and two-span station as an example, make $F_{i j}$ ( $i$ is the transverse member layer, $i=1 \sim 3$; $j$ is the number of vertical component columns, $j=1 \sim 4$ ) the equivalent inertial force on the junction $i j$ in the subway station member. The value is the product of the correction factor and half the sum of the mass of the member around the nodes; the formula is shown below:

$$
F_{i j}=k \cdot m_{i j}
$$

$m_{i j}$ is half the sum of the mass of the member around the nodes; $k$ is equivalent inertial force coefficient.

\section{Determination of Equivalent Inertial Force Coefficient $(k)$}

4.1. Influence Factor. Underground structure seismic responses are influenced by many factors, such as the structure forms, depth, foundation soil properties, soil profile, and groundwater seepage. For ease of calculation, only two main factors need to be considered: the influence of structure depth and foundation soil properties on $k$.

Suppose $k$ is the combined effect of the main factors, the fortification intensity associated with the basic seismic acceleration value is recorded as $k_{s}$, and buried depth correction factor is referred as $k_{h}$, and foundation soil correction factor is referred to as $k_{t}$; then, the expression of the equivalent inertia force coefficient $k$ can be written as

$$
k=k_{s} k_{h} k_{t},
$$

where earthquake acceleration basic value $k_{s}$ can be selected according to the fortification intensity from the corresponding study of [14], as shown in Table 1.

4.2. The Effect of Depth Factor Correction $\left(k_{h}\right)$. Depth effect coefficient of equivalent inertial force is a function of the station depth $z, k_{h}=f(z)$. $k_{h}$ reflects the relationship between the depth and the value of equivalent inertial force. A regional strata consists of four kinds of soft soil, including clay, muddy clay, silty clay, and silt. In the seismic response analysis, the nonlinear behavior of soil should be considered and the dynamic constitutive relation of soil can be described by Davidenkov model [15], which is

$$
\begin{aligned}
& \frac{G}{G_{\max }}=1-\left[\frac{\left(\gamma / \gamma_{0}\right)^{2 B}}{1+\left(\gamma / \gamma_{0}\right)^{2 B}}\right]^{A} \\
& \frac{\lambda}{\lambda_{\max }}=\left[1-\frac{G}{G_{\max }}\right]^{\beta},
\end{aligned}
$$

where $G$ and $G_{\max }$, respectively, are the dynamic shear modulus and the maximum dynamic shear modulus of soils; $\lambda$ and $\lambda_{\text {max }}$ are the dynamic damping ratio and the maximum dynamic damping ratio; $\gamma$ is the dynamic shear strain and $\gamma_{0}$ is reference dynamic shear strain; $A, B$, and $\beta$ are the fitting parameters. The test parameters and the correlation coefficients $R^{2}$ of the four kinds of soil in Table 2 can be 
TABLE 2: The fitting parameters and the correlation coefficients $R^{2}$.

\begin{tabular}{lcccccc}
\hline \multirow{2}{*}{ Fitting parameters } & \multicolumn{2}{c}{$G / G_{\max }$} & \multicolumn{2}{c}{$\lambda / \lambda_{\max }$} & $R^{2}$ & $R^{2}$ \\
\hline Clay (muddy clay) & $A$ & $B$ & $\gamma_{0}\left(10^{-4}\right)$ & 0.9954 & 1.3690 & 0.9814 \\
Silty clay & 0.5773 & 0.6487 & 20.4 & 0.9987 & 1.3185 & 0.9815 \\
Silt & 1.2046 & 0.4527 & 7.1 & 0.9994 & 1.2468 & 0.9796 \\
\hline
\end{tabular}

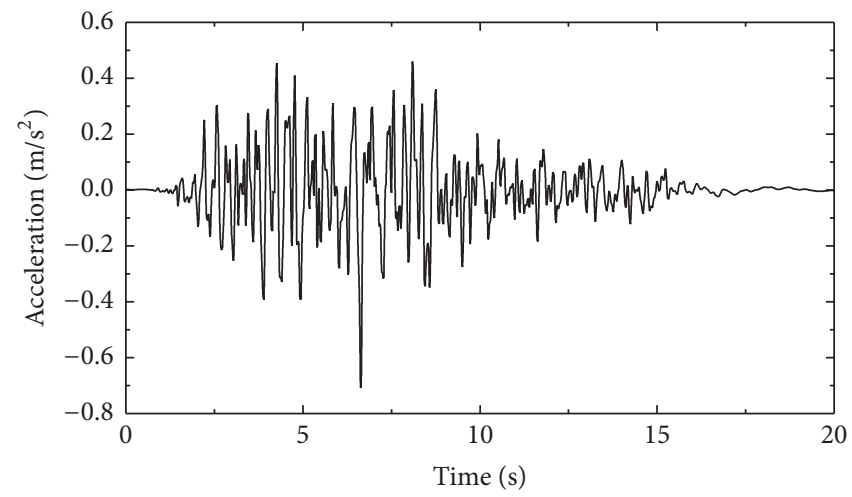

FIGURE 2: The synthetic horizontal seismic acceleration time-history wave of $70 \mathrm{~m}$ below ground surface within the exceeding probability $10 \%$ in 50 years.

obtained by soil dynamic tests [16]. The test results indicate that the dynamic characteristics of clay and muddy clay are similar, so their fitting parameters are same.

By using dynamic time-history analysis method and assuming that the soil is homogeneous, seismic response of structures is analyzed by taking the depth of $5 \mathrm{~m}, 10 \mathrm{~m}, 15 \mathrm{~m}$, $20 \mathrm{~m}, 25 \mathrm{~m}, 30 \mathrm{~m}$, and $35 \mathrm{~m}$ in the four kinds of soil. The soft soil in this region is more than two hundred meters thick, and it is difficult to use the bedrock input for seismic accelerations. Thus, the synthetic horizontal seismic acceleration time-history wave of $70 \mathrm{~m}$ below ground surface within the exceeding probability $10 \%$ in 50 years is inputted (Figure 2).

The points in Figure 3 are the maximum bending moment of the seismic response of subway station structures under different buried depth. Although the dynamic parameters of the clay and muddy clay are same in Table 2, there exist differences between maximum values of bending moment under the same buried depth, which is due to the difference of their static properties. The lines in Figure 3 are the fitting curves between the depth and the maximum bending moment. The correlation coefficients of clay, muddy clay, silty clay, and silt are $0.94677,0.98217,0.67003$, and 0.85064 , respectively, which shows good linear correlation. It can be seen that muddy clay shows the best linear fitting effect.

Engineering foundation is a complex of a variety of soils. To study the relationship between the structure depth and internal force in actual condition, the average value of the maximum bending moment of the four types of soil in subway station structure is taken to fit with depth. As shown in Figure 4, the depth and internal force remain linear correlation and the fitting relation is

$$
M=240.0-2.21 z \quad\left(R^{2}=0.9802\right),
$$

where $M$ is the average value of the maximum bending moment of subway station structure; $z$ is the net depth of subway station structure roof surface to the ground; 240.0 refers to the maximum value of the bending moment of the structure with a buried depth of $0 \mathrm{~m}$. To divide the fitting relation (4) by 240.0 , and the correction formula of buried depth buried depth is obtained:

$$
k_{h}=1-0.0093 z \text {. }
$$

4.3. The Effect of Foundation Soil Factor Correction $\left(k_{t}\right)$. Take the equivalent inertial force coefficient of the $i$-layer soil as $k_{t i}$. Equivalent inertia force influence coefficient of foundation soil is denoted as $k_{t}$. It reflects the combined effects of multilayer soils in the internal forces of the structure. The soil depth that has strong impact on dynamic internal force is recorded as $H$.

From the definition of $k_{t i}$, it is known that the value of $k_{t i}$ can be gotten according to the principle of equal maximum effect of dynamic time-history analysis and equivalent inertia force method. If the subway station is located in a single specie of the soil, subway station structures can be analyzed by using dynamic time-history method in the case of 0 depth $(z=0)$, and the same working conditions can be calculated according to equivalent inertial force diagram.

Make sure the maximum value of the structural moment calculated by the equivalent inertial force method is equal to that of the dynamic time-history analysis by adjusting the value of the equivalent inertial force. Since the structure depth is $0 \mathrm{~m}$ and buried depth correction factor $k_{h}$ is 1 , then $k$ is only related to a single soil property. In other words, the equivalent inertial force coefficient of the $i$-layer soil is $k_{t i}$. The value of various soils $k_{t i}$ is shown in Table 3. The 


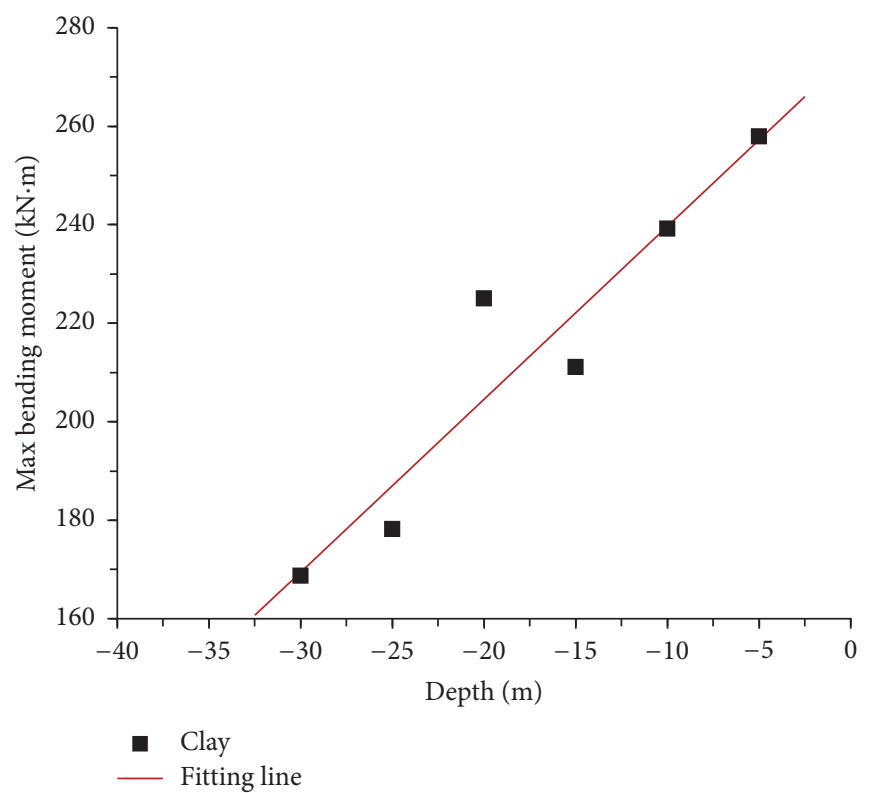

(a) Clay

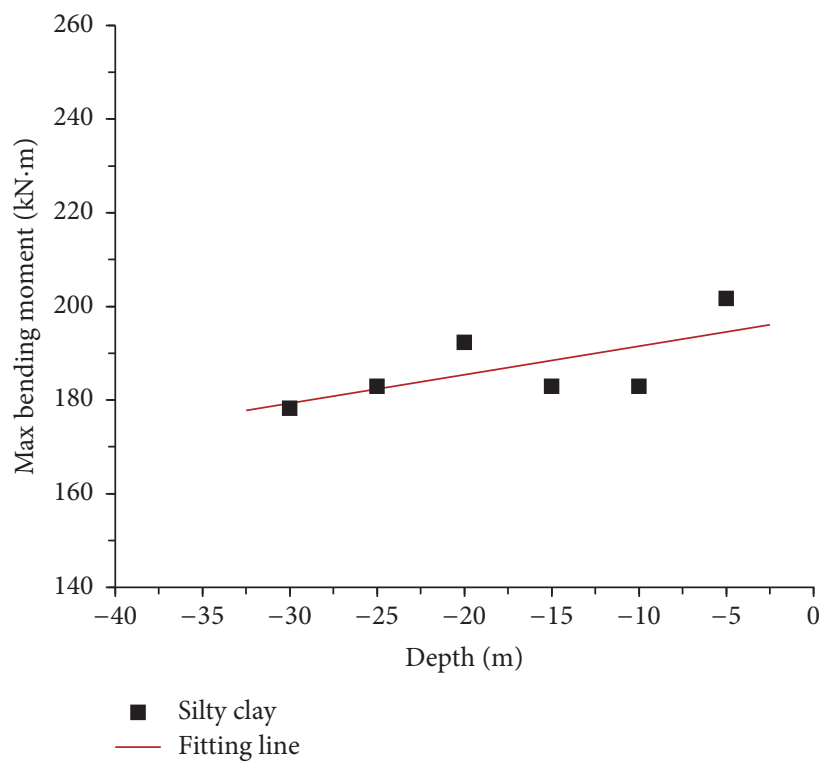

(c) Silty clay

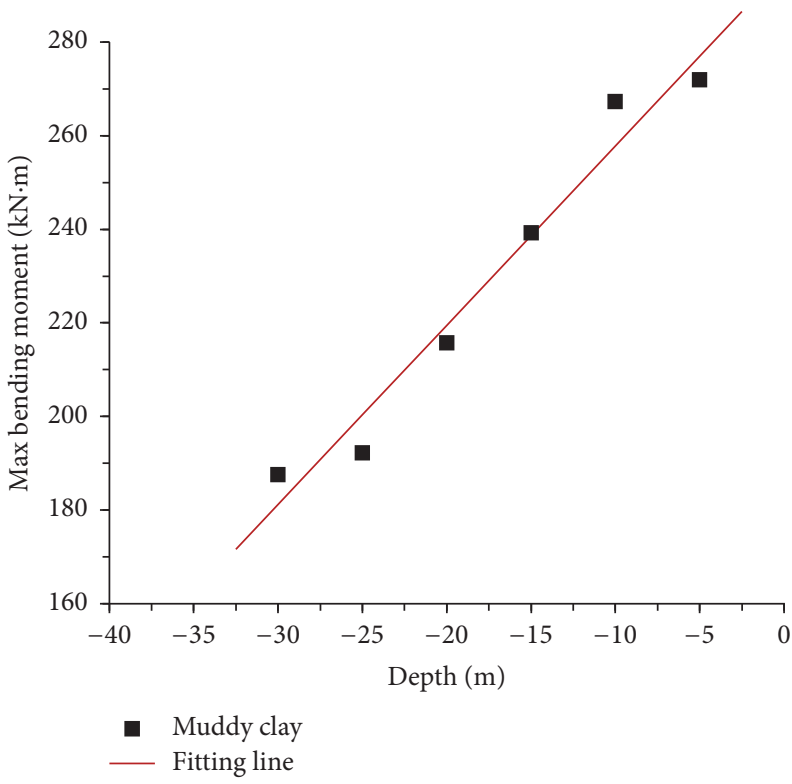

(b) Muddy clay

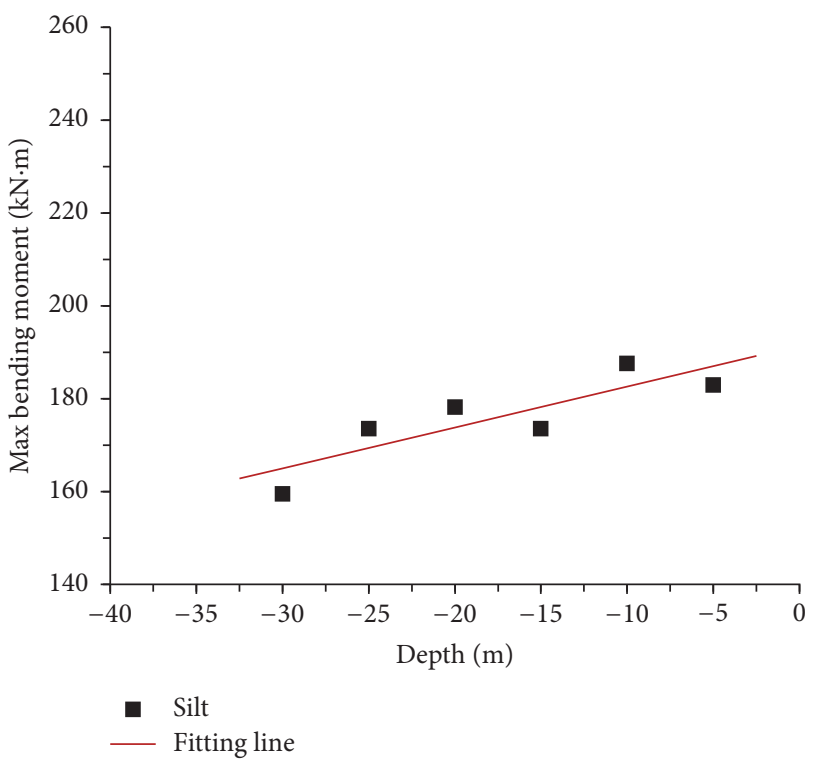

(d) Silt

FIGURE 3: The relationship between depth and maximum bending moment.

TABLE 3: The equivalent inertial force coefficient $\left(k_{t i}\right)$.

\begin{tabular}{lccc}
\hline Clay & Muddy clay & Silty clay & Silt \\
\hline 4.3 & 4.8 & 4.5 & 3.7 \\
\hline
\end{tabular}

difference of $k_{t i}$ between clay and muddy clay is also caused by the difference of static properties.

$k_{t}$ is the equivalent inertial force coefficient of the foundation soil and reflects the combined effects of internal forces of a variety of soils. Therefore, introduce method of weighted mean to calculate the data in Table 3 in terms of soil thickness of each layer and then obtain the influence coefficient of foundation soil surrounding subway station to the value of equivalent inertial force:

$$
k_{t}=f\left(k_{t i}\right)=\sum_{i=1}^{N} \frac{H_{i}}{H} k_{t i}
$$

where $N$ is the total number of soil layers that has big impact on the seismic response of subway station structure from the surface of soil; $H_{i}$ is the thickness of the $i$-layer of the soil; $H$ is the overall thickness of the soil that has greater impact on the seismic response of structures from the surface. According to dynamic time-history analysis of the research results, when the calculating area is taken as three times structure height 


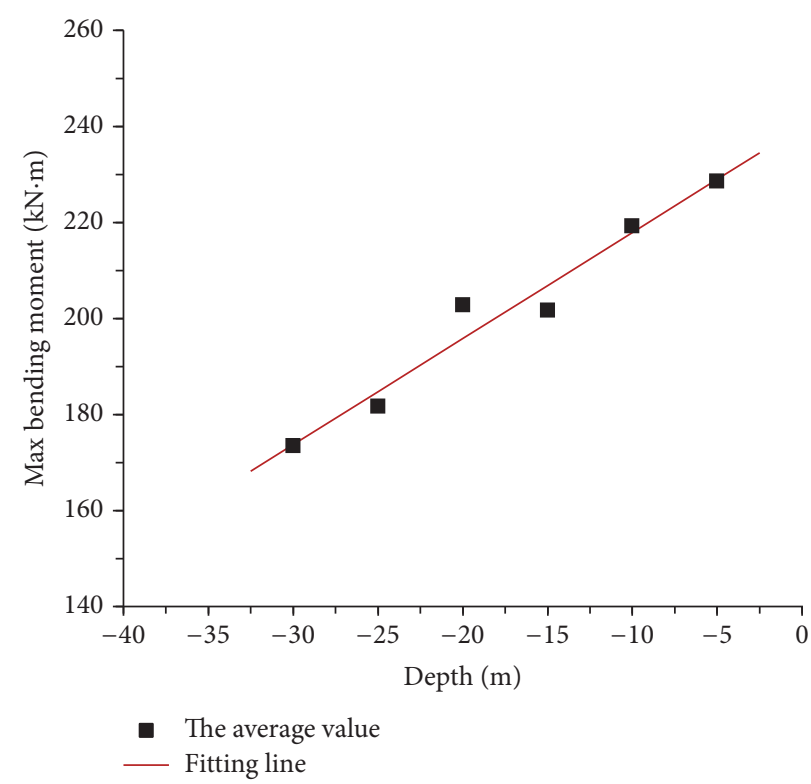

FIGURE 4: The relationship between the average value of the maximum bending moment and depth in subway station structure.

below the floor, the results will be stable. Therefore, it is recommended to take the $H$ three times the height of the structure from surface to bottom.

Above all, under 7-degree fortification intensity, the equivalent inertial force coefficient is expressed as below:

$$
k=k_{s} k_{h} k_{t}=0.1 g(1-0.0093 z) \sum_{i=1}^{N} k_{i i} .
$$

\section{Axial Force and Shear Force Correction}

The equivalent inertial force method is based on the principle that the maximum bending moment values obtained by equivalent inertial force method and dynamic time-history analysis method are equal and the location are same. The correction factor needs to be introduced to determine the bending moment of the remaining members as well as axial force and shear force of all the components to reach the same result with that of dynamic time-history analysis. To obtain the internal force correction coefficient of the equivalent inertial force, muddy clay and silty clay that have big differences of internal force in dynamic time-history analysis are selected and calculate the value in different depth; then, compare the results with equivalent inertial force to get the axial force and shear force correction. The result is shown in Table 4 .

Average the data in Table 4, and the value will be calculated according to $15 \%$ of the results compared with dynamic time-history analysis. Therefore, the range of internal force correction coefficient is obtained (Table 5).

\section{Example}

6.1. Design Information. For a certain city in this region, the structure needs to remain intact through the medium earthquake under the seismic fortification intensity. When seismic intensity is 7 degrees, the structure is in elastic stress state.
Sectional shape and geometry are shown in Figure 5, and the main dimensions are as follows: subway station width is $24.2 \mathrm{~m}$, height is $12.87 \mathrm{~m}$, cross-sectional dimension of upper and underlying column is $500 \mathrm{~mm} \times 1000 \mathrm{~mm}$, and their column space is $8.00 \mathrm{~m}$, the roof thick is $900 \mathrm{~mm}$ (depth is $3 \mathrm{~m}$ ), the thickness of medium plate is $400 \mathrm{~mm}$, and the floor thickness is $1100 \mathrm{~mm}$. Distribution of soil is shown in Figure 6, characteristic parameters of soil are shown in Table 6, and characteristic parameters of structure are shown in Table 7.

6.2. Computation Model. The dynamic internal forces of the station structure are calculated by using equivalent inertial force method and dynamic time-history analysis method. When using equivalent inertial force method, the calculation software is GeoFBA2D. The equivalent horizontal seismic inertial force at the nodal point is determined by formula (1), and internal forces of station structure can be amended by using the data listed in Table 5. When using dynamic timehistory analysis method, the calculation software is Flac2D. Mesh generation is shown in Figure 7. The width of the calculation area is $170 \mathrm{~m}$, where both sides of the structure are three times the width of the station. The depth of the calculation area is $70 \mathrm{~m}$, and the depth of soil from the bottom plate is about $54 \mathrm{~m}$, which is more than three times the height of the station. The bottom boundary of the calculation area is vertically fixed and horizontally free; the top boundary is free of deformation; the lateral boundaries are free-field, to ensure that all the waves on the boundaries are absorbed. The synthetic acceleration time-history wave of $70 \mathrm{~m}$ below ground surface within the exceeding probability $10 \%$ in 50 years is inputted from the bottom boundary (Figure 2).

6.3. Computation Result. The diagrams of bending moment, shear force, axial force of the maximum dynamic internal force of station structure according to equivalent inertial force method, and dynamic time-history method are shown 


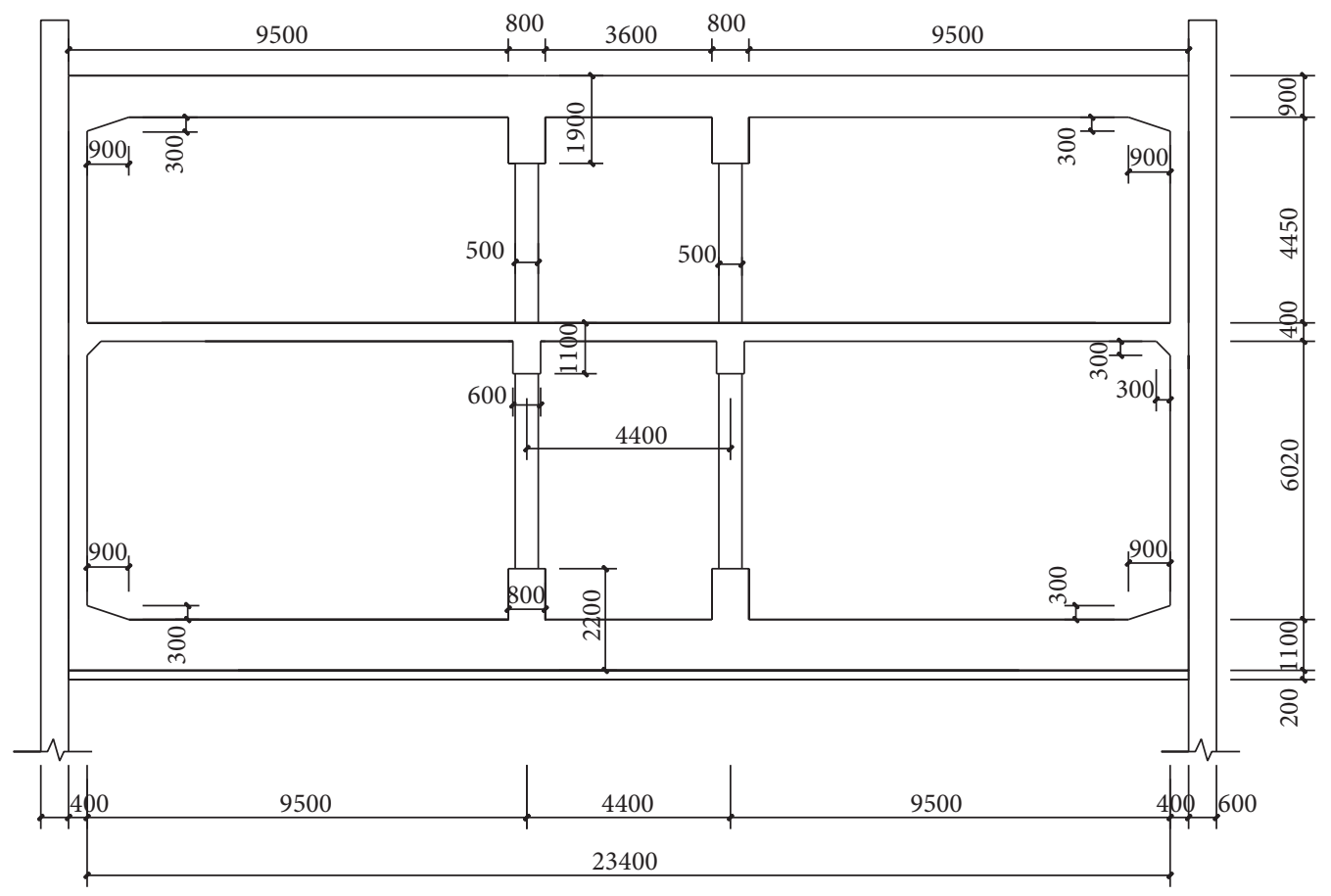

FIGURE 5: Sectional shape and geometry of two layers and three crosses subway station structure.

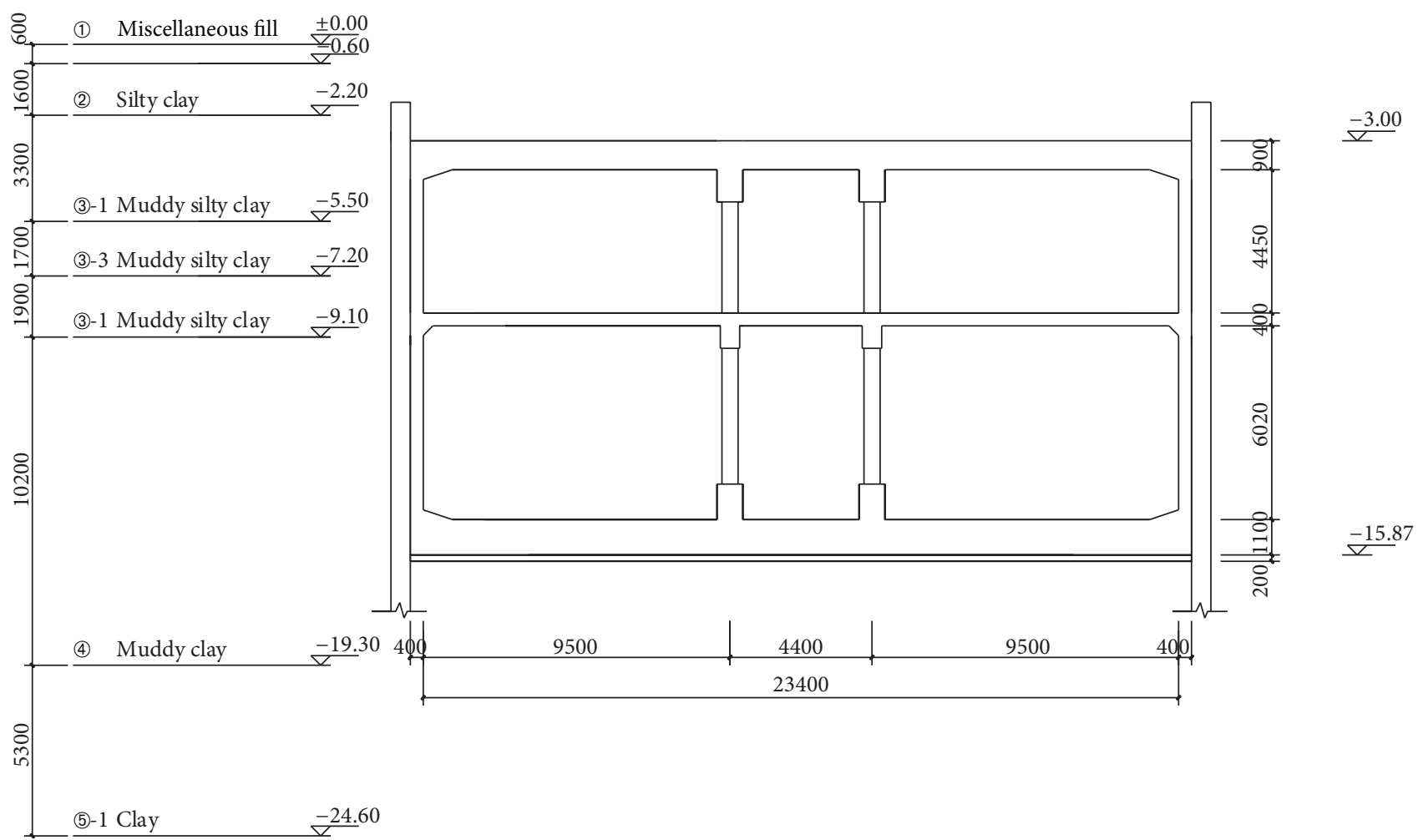

Figure 6: Soil distribution. 
TABLE 4: The internal force correction coefficient by using equivalent inertial force method.

\begin{tabular}{|c|c|c|c|c|c|c|c|}
\hline & Soil layer & Depth & Floor & Medium plate & Roof & Flank & Middle column \\
\hline \multirow{4}{*}{ Bending moment } & \multirow{2}{*}{ Muddy clay } & $1 \mathrm{~m}$ & 1.00 & 0.59 & 0.34 & 0.70 & 0.53 \\
\hline & & $3 \mathrm{~m}$ & 1.00 & 0.54 & 0.43 & 0.70 & 0.56 \\
\hline & \multirow{2}{*}{ Silty clay } & $1 \mathrm{~m}$ & 1.00 & 0.33 & 0.21 & 0.70 & 0.25 \\
\hline & & $3 \mathrm{~m}$ & 1.00 & 0.36 & 0.28 & 0.70 & 0.28 \\
\hline \multirow{4}{*}{ Shear force } & \multirow{2}{*}{ Muddy clay } & $1 \mathrm{~m}$ & 1.83 & 0.88 & 0.47 & 0.31 & 0.55 \\
\hline & & $3 \mathrm{~m}$ & 1.64 & 0.89 & 0.80 & 0.30 & 0.55 \\
\hline & \multirow{2}{*}{ Silty clay } & $1 \mathrm{~m}$ & 2.36 & 0.49 & 0.40 & 0.46 & 0.28 \\
\hline & & $3 \mathrm{~m}$ & 1.33 & 0.52 & 0.84 & 0.47 & 0.30 \\
\hline \multirow{4}{*}{ Axial force } & \multirow{2}{*}{ Muddy clay } & $1 \mathrm{~m}$ & 0.50 & 0.05 & 1.75 & 1.91 & 0.78 \\
\hline & & $3 \mathrm{~m}$ & 0.50 & 0.05 & 2.67 & 1.88 & 1.29 \\
\hline & \multirow{2}{*}{ Silty clay } & $1 \mathrm{~m}$ & 0.87 & 0.07 & 2.09 & 3.03 & 0.93 \\
\hline & & $3 \mathrm{~m}$ & 0.87 & 0.06 & 3.27 & 3.07 & 1.16 \\
\hline
\end{tabular}

TABLE 5: The range of internal force correction coefficient on the basis of the equivalent inertial force method.

\begin{tabular}{|c|c|c|c|c|c|c|}
\hline & & Floor & Medium plate & Roof & Flank & Middle column \\
\hline \multirow{2}{*}{ Bending moment } & Value & 1.00 & $0.36-0.52$ & $0.24-0.34$ & $0.60-0.86$ & $0.31-0.45$ \\
\hline & Interval & 0 & 0.16 & 0.11 & 0.26 & 0.14 \\
\hline \multirow{2}{*}{ Shear force } & Value & $1.46-2.10$ & $0.60-0.85$ & $0.51-0.74$ & $0.31-0.42$ & $0.36-0.52$ \\
\hline & Interval & 0.64 & 0.25 & 0.23 & 0.11 & 0.16 \\
\hline \multirow{2}{*}{ Axial force } & Value & $0.55-0.78$ & $0.05-0.08$ & $1.90-2.73$ & $2.02-2.90$ & $0.82-1.16$ \\
\hline & Interval & 0.23 & 0.03 & 0.83 & 0.87 & 0.34 \\
\hline
\end{tabular}

TABLE 6: Soil characteristics parameter.

\begin{tabular}{|c|c|c|c|c|c|c|c|}
\hline Number & Name of soil layer & Depth (m) & $\begin{array}{l}\text { Unit weight } \\
\left(\mathrm{kN} / \mathrm{m}^{3}\right)\end{array}$ & $\begin{array}{c}\text { Cohesion } \\
(\mathrm{kPa})\end{array}$ & $\begin{array}{l}\text { Angle of internal } \\
\text { friction (degree) }\end{array}$ & $\begin{array}{c}\text { Average shear wave } \\
\text { velocity }(\mathrm{m} / \mathrm{s})\end{array}$ & $\begin{array}{c}\text { Compression } \\
\text { modulus }(\mathrm{MPa})\end{array}$ \\
\hline (1) & Miscellaneous fill & 0.6 & 18.4 & & & & \\
\hline (2) & Silty clay & 1.6 & 18.5 & 15 & 28 & 115 & 3.3 \\
\hline (3) -1 & Muddy silty clay & 3.3 & 17.5 & 11 & 18 & 120 & 4.1 \\
\hline (3) -3 & Muddy silty clay & 1.7 & 18.5 & 4 & 29.5 & 125 & 5.0 \\
\hline (3)-1 & Muddy silty clay & 1.9 & 17.5 & 11 & 18 & 125 & 5.2 \\
\hline (4) & Muddy clay & 10.2 & 16.6 & 14 & 11 & 140 & 2.4 \\
\hline (5)- 1 & Clay & 5.3 & 17.4 & 15 & 12.5 & 175 & 5.0 \\
\hline
\end{tabular}

TABLE 7: Physical and mechanical parameters of concrete.

\begin{tabular}{lccc}
\hline Material & Density $\left(\mathrm{kg} / \mathrm{m}^{3}\right)$ & Modulus of elasticity $(\mathrm{MPa})$ & Poisson ratio \\
\hline Concrete C35 & $2.5 \times 10^{3}$ & $3.2 \times 10^{4}$ & 0.25 \\
\hline
\end{tabular}

in Figures 8-10. The maximum internal force of subway station structure is shown in Table 8.

On the reinforcement calculation of structures, usually the maximum internal force of section is the most concerned. Through the above analysis, we can know that the results of the maximum bending moment by using equivalent seismic inertial force method are the same as those of using dynamic time-history analysis method. The maximum relative error of each member is less than $10 \%$, and appears in the same site. The maximum shear forces of two methods emerge in the same site and the relative errors are within $10 \%$. The maximum axial forces of two methods also emerge in the same site and the relative errors of the members are within $10 \%$ except for the small axial forces in middle column.

\section{Conclusion}

(1) The maximum shear force, axial force, and bending moment by using equivalent seismic inertial force method appear in the same place compared with the results of dynamic time-history analysis method, and the relative errors can be controlled within $10 \%$. 


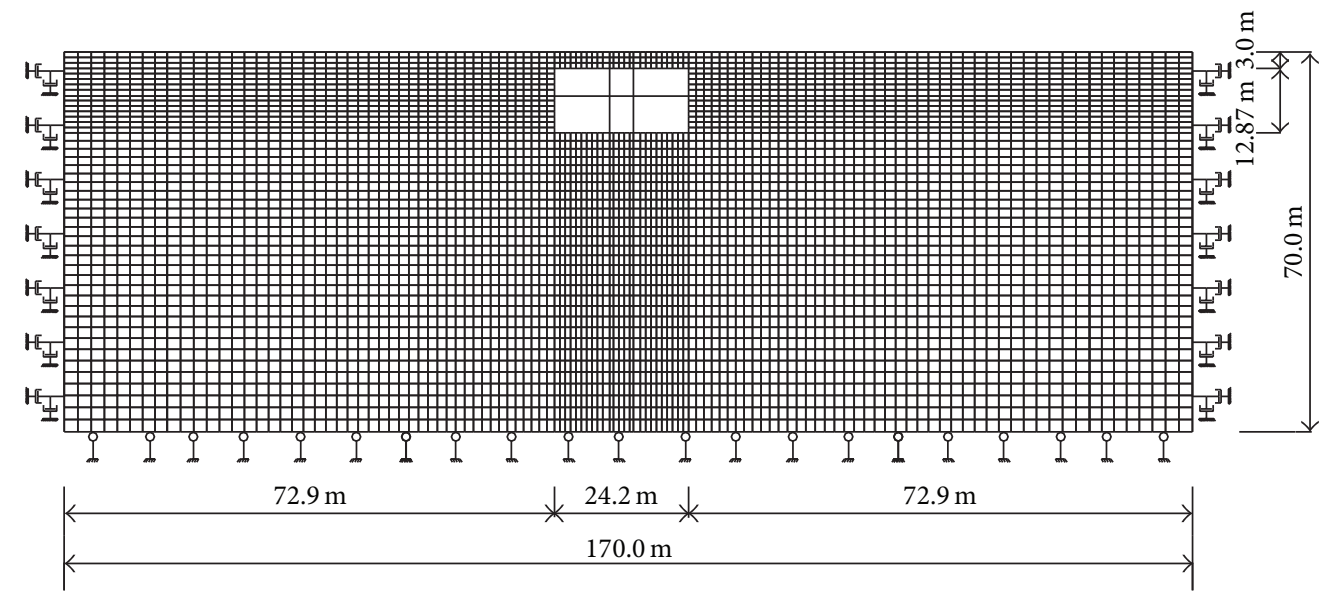

FIGURE 7: The scope of the calculation area and the mesh generation map.

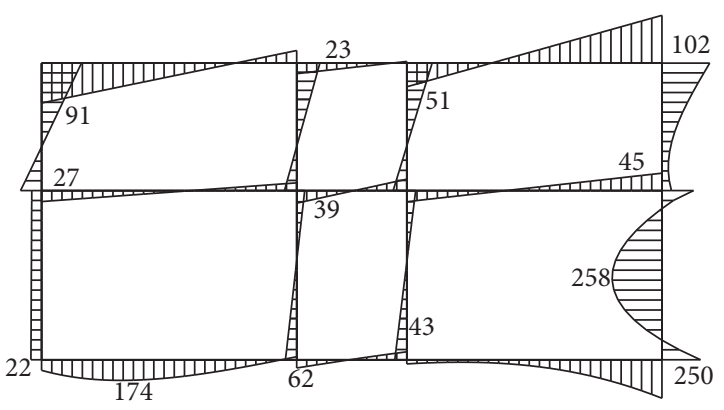

(a) Equivalent inertial force method

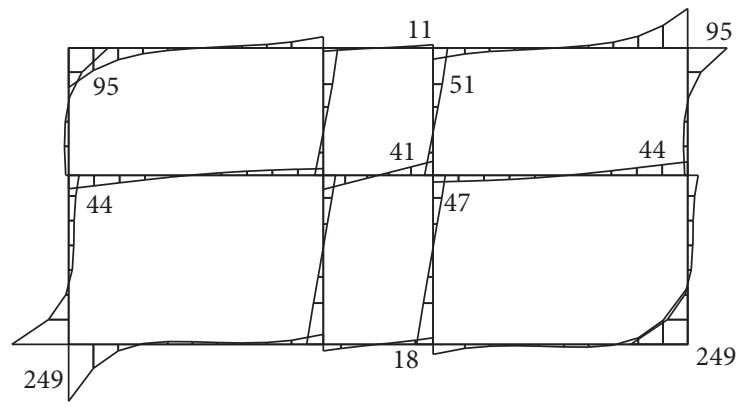

(b) Dynamic time-history method

FIGURE 8: The moment diagram of the maximum dynamic internal force of two layers and three crosses station structure (kN.m).

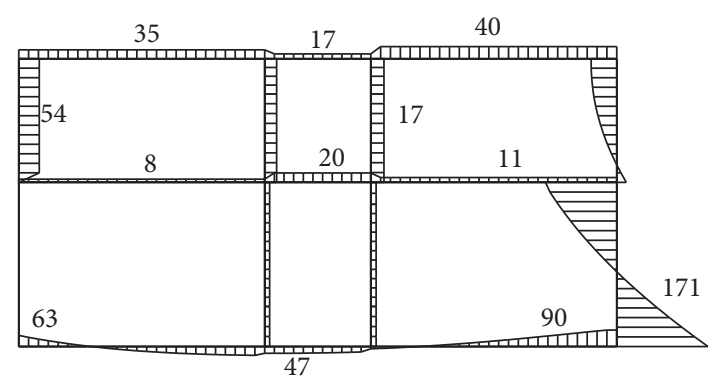

(a) Equivalent inertial force method

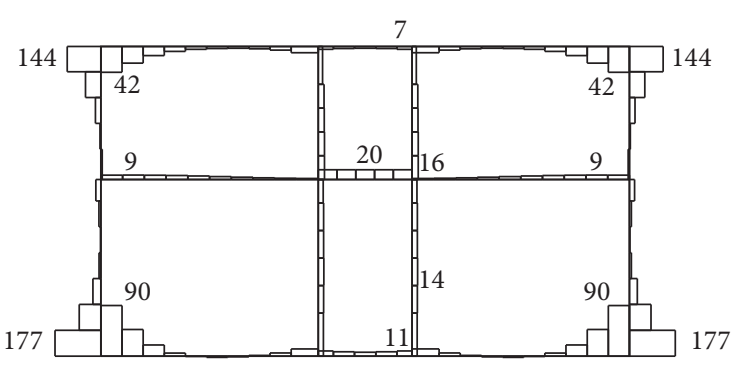

(b) Dynamic time-history method

Figure 9: The shear force diagram of the maximum dynamic internal force of two layers and three crosses station structure (kN).

TABLE 8: The maximum dynamic internal force of station structure according to equivalent inertial force method and dynamic time-history method.

\begin{tabular}{lcccccc}
\hline & \multicolumn{2}{c}{ Equivalent inertial force method } & \multicolumn{3}{c}{ Dynamic time-history method } \\
& $\begin{array}{c}\text { Bending moment } \\
(\mathrm{kN} \cdot \mathrm{m})\end{array}$ & $\begin{array}{c}\text { Shear force } \\
(\mathrm{kN})\end{array}$ & $\begin{array}{c}\text { Axial force } \\
(\mathrm{kN})\end{array}$ & $\begin{array}{c}\text { Bending moment } \\
(\mathrm{kN} \cdot \mathrm{m})\end{array}$ & $\begin{array}{c}\text { Shear force } \\
(\mathrm{kN})\end{array}$ & $\begin{array}{c}\text { Axial force } \\
(\mathrm{kN})\end{array}$ \\
\hline Floor & 250 & 90 & 280 & 249 & 90 & 278 \\
Medium plate & 45 & 20 & 17 & 44 & 20 & 18 \\
Roof & 102 & 40 & 126 & 249 & 42 & 126 \\
Flank & 258 & 171 & 214 & 51 & 37 & 215 \\
Middle column & 51 & 17 & 32 & 33 \\
\hline
\end{tabular}




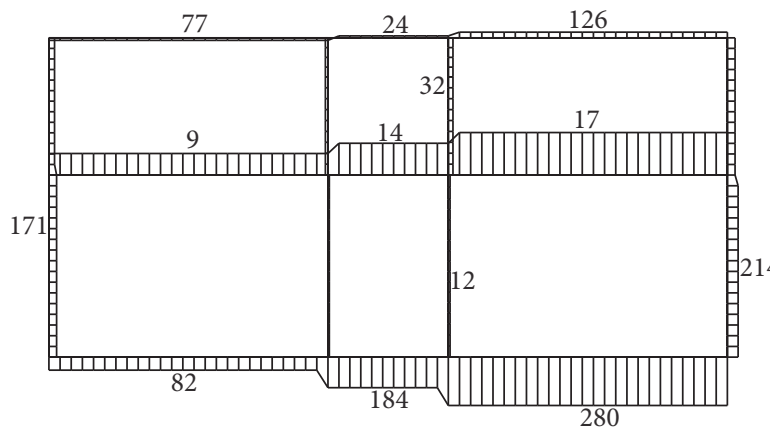

(a) Equivalent inertial force method

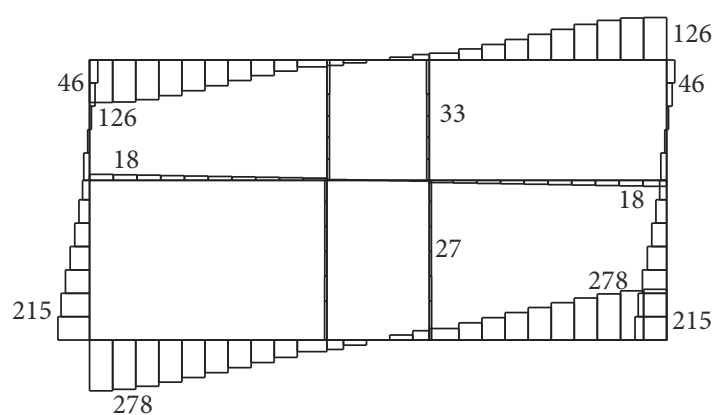

(b) Dynamic time-history method

FIGURE 10: The axial force diagram of the maximum dynamic internal force of two layers and three crosses station structure (kN).

(2) Through adjusting the seismic inertia force coefficient and internal force correction factor, seismic calculation method of soft soil stratum in subway station can be established on basis of equivalent seismic inertial force method. This method can be a simple way used for seismic design.

\section{Competing Interests}

The authors declare that there is no conflict of interests regarding the publication of this paper.

\section{References}

[1] Y. M. A. Hashash, J. J. Hook, B. Schmidt, and J. I-Chiang Yao, "Seismic design and analysis of underground structures," Tunnelling and Underground Space Technology, vol. 16, no. 4, pp. 247-293, 2001.

[2] S.-H. Nam, H.-W. Song, K.-J. Byun, and K. Maekawa, "Seismic analysis of underground reinforced concrete structures considering elasto-plastic interface element with thickness," Engineering Structures, vol. 28, no. 8, pp. 1122-1131, 2006.

[3] H. Huo, A. Bobet, G. Fernández, and J. Ramírez, "Analytical solution for deep rectangular structures subjected to far-field shear stresses," Tunnelling and Underground Space Technology, vol. 21, no. 6, pp. 613-625, 2006.

[4] J. Li, Seismic Response Analysis of Underground Space of WuHan, Department of Civil Engineering and Architecture, Wuhan University of Technology, WuHan, China, 2010.

[5] X.-W. Tang, P.-S. Fu, H. Li, and J.-F. Gong, "Seismic simulation and analysis of the two-layer prestressed long-span subway station," Journal of Northeastern University (Natural Science), vol. 36, no. 6, pp. 893-896, 2015.

[6] Q. I. Hongwei, "Application of response displacement method in seismic calculation of the Subway," Railway Construction Technology, vol. S2, pp. 100-102, 2012.

[7] Y. Linde, J. Qianqian, Z. Yonglai, and Y. Chao, "Shaking table test on metro station structures in soft soil," Modern Tunnelling Technology, vol. 40, no. 1, pp. 7-11, 2003.

[8] H.-Y. Zhuang, S.-G. Cheng, and G.-X. Chen, "Numerical simulation and analysis of earthquake damages of Dakai metro station caused by Kobe earthquake," Rock and Soil Mechanics, vol. 29, no. 1, pp. 245-250, 2008.
[9] P. Liu, "Subway station seismic dynamic finite element response calculation in considering soil-structure interaction," Architectural Engineering Technology and Design, vol. 34, pp. 323-324, 2014.

[10] Y. Huan, Q. Fang, J.-C. Liu, L. Chen, and M.-L. Du, "Theoretical and numerical investigations on enhancement of aseismic capability of metro stations," Journal of Vibration and Shock, vol. 30, no. 3, pp. 253-257, 2011.

[11] J.-J. Lv and Z.-Q. Zhao, "Seismic analysis of typical subway station structure of Tianjin Metro Line 10," Journal of Railway Engineering Society, vol. 32, no. 10, pp. 126-130, 2015.

[12] D. Quan, Y. Wang, Y. Jing et al., "Review of anti-seismic structures of subway in Loess Area," Technology for Earthquake Disaster Prevention, vol. 10, no. 3, pp. 566-574, 2015.

[13] Code for Geotechnical Investigation of Urban Rail Transit GB50307-2012, China Planning Press, Beijing, China, 2012.

[14] GB, "Code for seismic design of buildings," Tech. Rep. GB500112010, China Building Industry Press, Beijing, China, 2010.

[15] P. P. Martin and H. B. Seed, "One-dimensional dynamic ground response analyses," Journal of the Geotechnical Engineering Division, vol. 108, no. 7, pp. 935-952, 1982.

[16] Q. Liu, Study on Seismic Design and Analysis Theory for Subway Structures in Soft Soil, School of Civil Engineering, Tongji University, Shanghai, China, 2005. 


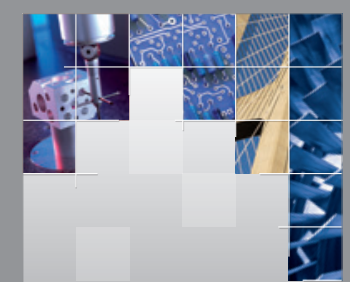

\section{Enfincering}
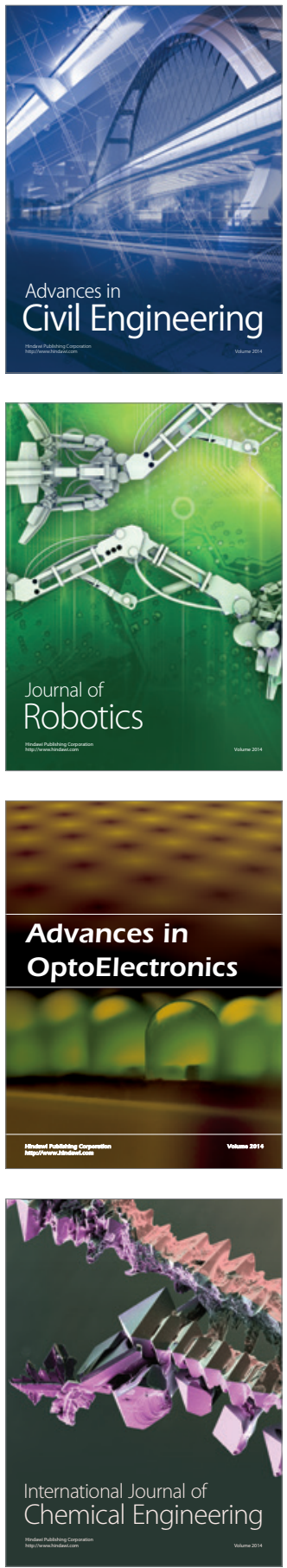

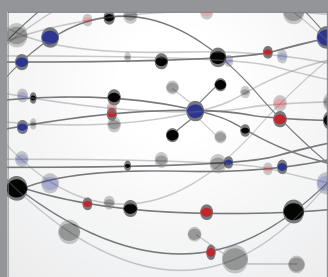

The Scientific World Journal

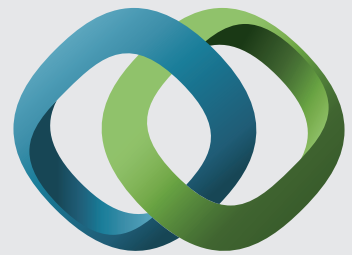

\section{Hindawi}

Submit your manuscripts at

http://www.hindawi.com
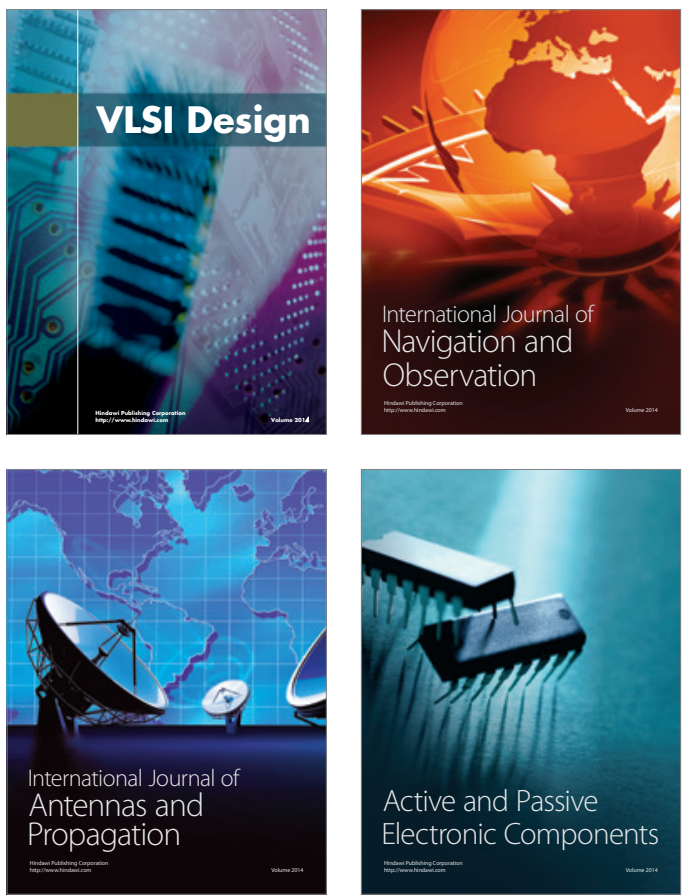
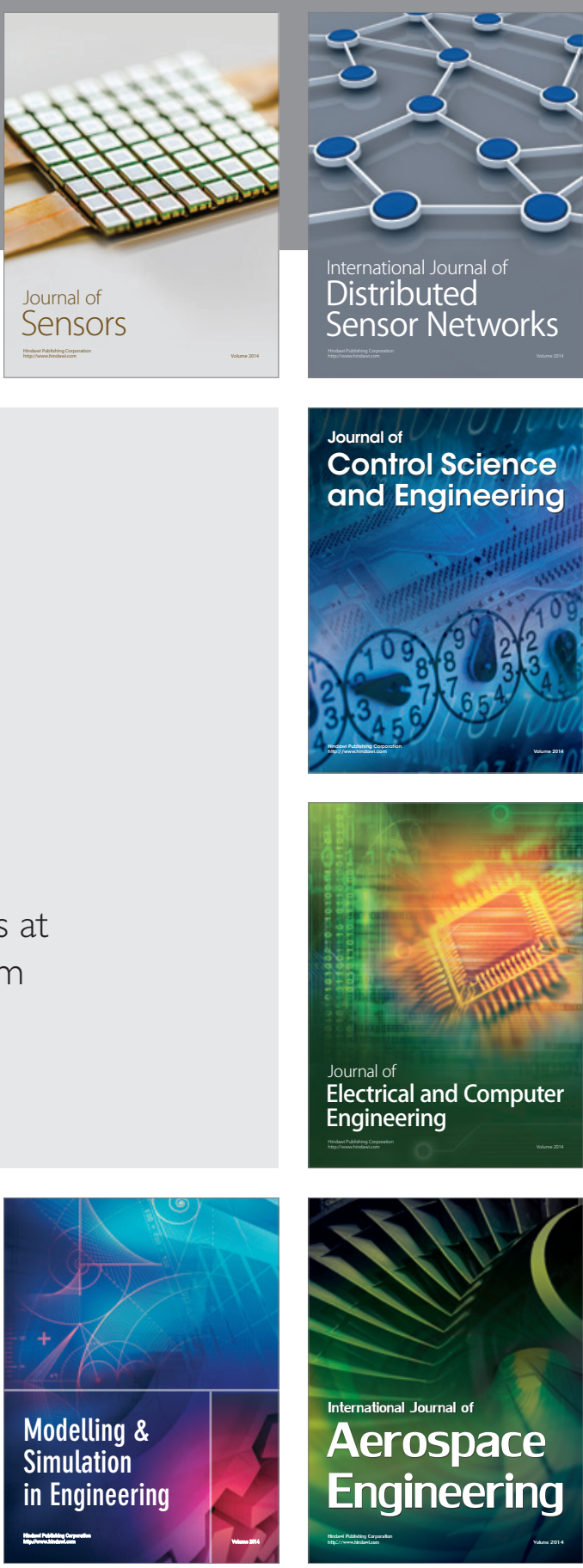

International Journal of

Distributed

Sensor Networks

Journal of

Control Science

and Engineering
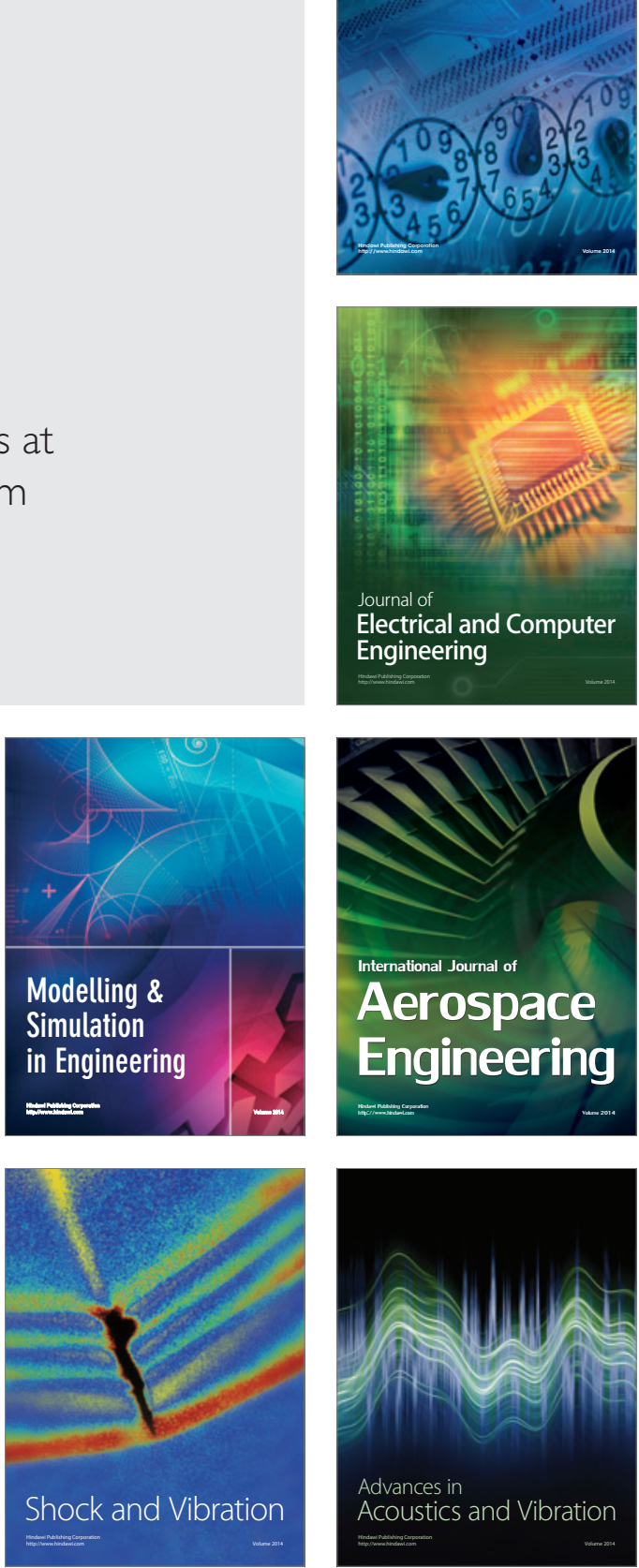